\title{
Programa de Intervenção Fonológica Associado à Correspondência Grafema-Fonema em Escolares de Risco para a Dislexia
}

\author{
Phonological Intervention Program Associated with Grapheme-Phoneme \\ Correspondence in Students at Risk for Dyslexia
}

\author{
Maryse Tomoko Matsuzawa Fukuda \& Simone Aparecida Capellini* \\ Universidade Estadual Paulista (UNESP), Marília, Brasil
}

\begin{abstract}
Resumo
Este estudo objetivou identificar os escolares com sinais de risco para a dislexia e verificar a eficácia do programa de treinamento fonológico associado à correspondência grafema-fonema nessa população. Participaram deste estudo 60 escolares divididos em: GI, composto por 30 escolares submetidos ao programa de treinamento, e GII, composto por 30 escolares não submetidos ao programa de treinamento. Os resultados revelaram que, após o período de realização do programa de treinamento, o GI apresentou diferenças estatisticamente significantes em todas as habilidades propostas na avaliação. Conclui-se que o programa de treinamento das habilidades fonológicas e correspondência grafema-fonema foi eficaz para a identificação dos escolares com sinais de dislexia, comprovando-se pela melhora das habilidades cognitivo-linguísticas na situação de pós-testagem em relação à pré-testagem.
\end{abstract}

Palavras-chave: Dislexia, leitura, avaliação, consciência fonológica.

\begin{abstract}
This study aimed to identify students at risk for dyslexia and verify the efficacy of the phonological training and grapheme-phoneme correspondence program with such students. Sixty students participated in the study. They were divided into GI, consisting of 30 students who were submitted to the training program, and GII, consisting of 30 students who were not submitted to the training program. The results showed that the GI present statistically significant differences in all skills proposed in the evaluation after the training program. It is concluded that the phonological ability training and grapheme-phoneme correspondence program were effective for the identification of students with signs of dyslexia. It was proven by the improvement of the phonological abilities of reading in the post-testing situation compared to pre-testing.

Keywords: Dyslexia, reading, evaluation, phonological awareness.
\end{abstract}

As habilidades básicas como ler e escrever são importantes não apenas para a aprendizagem escolar, mas, principalmente, para a vida fora da escola. Os fracassos na aquisição e desenvolvimento dessas habilidades tendem a ocasionar desinteresse, baixo desempenho e, com frequência, evasão escolar (Marinotti, 2004).

Ao serem realizadas investigações sobre as possíveis causas dos problemas de aprendizagem nos escolares, deve-se ter o cuidado de diferenciar aqueles que apresentam dificuldades de origem social-econômica-cultural e emocional daqueles que apresentam alterações nas

\footnotetext{
"Agradecimentos: Ao Conselho Nacional de Desenvolvimento Científico e Tecnológico (CNPq) pela concessão da bolsa de produtividade em pesquisa para a segunda autora. Endereço para correspondência: Avenida Hygino Muzzi Filho, 737, Marília, SP, Brasil 17525-900. E-mail:sacap@uol.com.br
}

habilidades cognitivo-linguísticas de origem genéticoneurológica, como a dislexia do desenvolvimento e o distúrbio de aprendizagem (Capellini \& Ciasca, 2000; Capellini, Padula, \& Ciasca, 2004; Capellini \& Salgado, 2003).

Em relação à dislexia, a World Federation of Neurology, em 1968, definiu-a como sendo um transtorno de aprendizagem da leitura que ocorre apesar de inteligência normal, de ausência de problemas sensoriais ou neurológicos, de instrução escolar e oportunidades socioculturais adequadas e suficientes, além disso, depende da existência de perturbação de aptidões cognitivas fundamentais, frequentemente de origem constitucional (Critchley, 1985).

A dislexia é caracterizada pela dificuldade no processamento da linguagem, manifestando-se primeiramente no processamento fonológico da fala e, posteriormente, no pro- 
cessamento fonológico envolvido na leitura. Sua prevalência é de 5 a 17\% na população (Temple et al., 2000).

Em decorrência do déficit fonológico, algumas pesquisas realizadas, desde a década de 80 , descrevem a necessidade da realização de programas de intervenção, também conhecidos internacionalmente como programas de remediação por enfatizarem o ensino da relação letrasom e das habilidades metalinguísticas necessárias para a aprendizagem do sistema de escrita com base alfabética (Capellini et al., 2010).

O presente estudo teve como base a pesquisa desenvolvida por Schneider, Roth e Ennemoser (2000), que recomendam o uso da intervenção em crianças que apresentam os sinais da dislexia logo nas séries iniciais de alfabetização. Este estudo teve como objetivo verificar se, após a realização de programas específicos, com as habilidades cognitivo-linguísticas alteradas, os escolares apresentam melhora para a aprendizagem da leitura ou se eles permanecem com a defasagem nessas habilidades, $o$ que poderia significar que os escolares realmente apresentam uma desordem de origem genético-neurológica que compromete a aquisição e o desenvolvimento de habilidades perceptivas e linguísticas. Alguns estudos internacionais (Agnew, Dorn, \& Eden, 2004; Elbro \& Petersen, 2004) recomendam que crianças que não apresentem melhora na aprendizagem de habilidades cognitivo-linguísticas após realização de programa de intervenção sejam submetidas a avaliações interdisciplinares para a investigação da existência do real quadro de dislexia. A falta de resposta à intervenção, segundo esses autores, é um critério de diagnóstico para a dislexia.

Segundo Abreu e Cardoso-Martins (1998), o conhecimento do nome das letras induz a criança a aprender a ler por meio do processamento parcial das relações letra-som nas palavras. Constatam que as crianças que conheciam os nomes das letras aprenderam a ler as grafias fonéticas mais facilmente do que as grafias visuais. Snow e Juel (2005) afirmam que a base fônica pode ser útil para as crianças não somente devido à relação letra-som, mas por fazer uma aproximação, dando à criança a oportunidade para descobrir o princípio alfabético. Além disso, ela oferece, na prática, o fechamento da ortografia da palavra.

Com base no exposto, este estudo objetivou identificar os escolares com sinais de risco para a dislexia e verificar neles a eficácia de um programa de treinamento das habilidades fonológicas associado à correspondência grafema-fonema.

\section{Método}

Este estudo se caracteriza por ser experimental e longitudinal e foi realizado com escolares de ensino público municipal da cidade de Marília-SP.

Dele participaram 60 escolares, de ambos os gêneros e na faixa etária entre 6 e 7 anos e 11 meses de idade, submetidos à mesma metodologia de alfabetização, ou seja, com enfoque sócio-construtivista (Tabela 1).
Tabela 1

Distribuição dos Escolares Submetidos à Avaliação para a Identificação dos Sinais da Dislexia Segundo Gênero

\begin{tabular}{|c|c|c|}
\hline Grupos & Masculino & Feminino \\
\hline & $N \quad(\%)$ & $N \quad(\%)$ \\
\hline GI & $18(30 \%)$ & $12(20 \%)$ \\
\hline GII & $14(23 \%)$ & $16(27 \%)$ \\
\hline
\end{tabular}

Como critérios de inclusão foram considerados a assinatura do Termo de Consentimento Livre e Esclarecido e a ausência de queixa auditiva, visual, cognitiva ou motora, descritas nos prontuários escolares ou por observação do professor. Como critérios de exclusão foram considerados a não assinatura dos pais ou responsáveis pelos escolares no Termo de Consentimento Livre e Esclarecido e a presença de deficiência sensorial, motora ou cognitiva descrita em prontuário escolar ou por observação do professor.

A identificação do risco para a dislexia nos escolares deste estudo foi realizada a partir da elaboração de provas de avaliação descritas na pesquisa original sobre treinamento de habilidades fonológicas e conhecimento de letra-som para esses escolares (Schneider et al., 2000), as quais foram normatizadas para a realidade brasileira por Capellini et al. (2009).

Inicialmente, então, todos os escolares participantes foram submetidos aos seguintes procedimentos:

Termo de Consentimento Livre e Esclarecido. Conforme resolução do Conselho Nacional de Saúde (CNS 196/ 96), anteriormente ao início do estudo, os pais ou responsáveis pelos escolares selecionados assinaram o Termo de Consentimento, autorizando a aplicação dos procedimentos.

Protocolo de Identificação Precoce dos Problemas de Leitura. Foram elaboradas sete provas para a identificação precoce dos problemas de leitura, com base na descrição da pesquisa original que oferece suporte teórico para sua realização. As provas aplicadas e que compõem o protocolo estão descritas a seguir:

1. Conhecimento do alfabeto. Foi apresentado ao escolar o alfabeto para que ele identificasse o nome da letra e o valor sonoro de cada letra apresentada.

2. Consciência fonológica. Prova composta de subtestes de produção de rima, identificação de rima, segmentação silábica, produção de palavras a partir do fonema dado, síntese fonêmica, análise fonêmica e identificação de som inicial. (a) Produção de rima: foram apresentadas, auditivamente, ao escolar 20 palavras, e solicitado que dissesse uma palavra que terminasse com o mesmo som. (b) Identificação de rima: foram apresentados, auditivamente, ao escolar 20 grupos de três palavras cada um, e solicitado que identificasse as palavras que terminassem com o mesmo som. (c) Segmentação silábica: foram apre- 
Fukuda, M. T. M \& Capellini, S. A. (2012). Programa de Intervenção Fonológica Associado à Correspondência Grafema-Fonema em Escolares de Risco para a Dislexia.

sentadas, auditivamente, ao escolar 21 palavras (dissílabas, trissílabas e quadrissílabas), e solicitado que separasse as palavras por número de sílabas. (d) Produção de palavras a partir do fonema dado: foram apresentados ao escolar os sons do alfabeto, e solicitado que dissesse uma palavra que começasse com o mesmo som. (e) Síntese fonêmica: foram apresentadas, auditivamente, ao escolar 21 palavras separadas por sons, e solicitado que dissesse a palavra formada. (f) Análise fonêmica: foram apresentadas, auditivamente, ao escolar 21 palavras e, em segui$\mathrm{da}$, solicitado que dissesse os sons de cada letra das palavras apresentadas. (g) Identificação de som inicial: foram apresentadas, auditivamente, ao escolar 21 palavras, e solicitado que dissesse o som inicial da primeira letra de cada palavra apresentada.

3. Memória de trabalho. Foram apresentadas, auditivamente, ao escolar 24 pseudopalavras, e solicitado que repetisse como havia entendido.

4. Velocidade de acesso à informação fonológica. Foram apresentadas ao escolar 7 sequências intercaladas de desenhos coloridos (carro, bola, pato, casa e chave), e solicitado que realizasse sua nomeação rápida.

5. Atenção visual. Foram apresentadas ao escolar 10 figuras coloridas para que ele identificasse entre duas palavras aquela que correspondesse a cada figura.

6. Leitura de palavras e pseudopalavras. Foram apresentadas, visualmente, ao escolar 40 palavras (20 palavras e 20 pseudopalavras) e solicitado para que realizasse a leitura em voz alta.

7. Compreensão de frases a partir de figuras apresentadas. Foram apresentadas ao escolar 20 frases incompletas e com figuras ilustrativas, e solicitado que as observasse e completasse as frases oralmente.

A aplicação desse procedimento foi realizado individualmente em apenas uma sessão com duração média de 50 minutos durante o horário de aula dos escolares, com a anuência e autorização da professora e da direção da escola em que foi realizado o estudo.

O material linguístico utilizado para a elaboração das provas descritas foi retirado de um banco de palavras de livros didáticos de $1^{\mathrm{a}}$ a $4^{\mathrm{a}}$ série utilizados na rede municipal de ensino de Marília-SP. Esclareça-se que as palavras utilizadas para a elaboração das provas seguem as regras de decodificação do português brasileiro tanto para palavras como para as pseudopalavras: (a) regra de correspondência grafofonêmica independente do contexto e (b) regra de correspondência grafofonêmica dependente do contexto (Scliar-Cabral, 2003). Levou-se em consideração, para a elaboração das provas, a seleção de palavras de alta frequência presentes em todos os livros de $1^{\mathrm{a}}$ a $4^{\mathrm{a}}$ série para que o estímulo linguístico não interferisse no desempenho do escolar.

A caracterização do desempenho dos 60 escolares deste estudo no Protocolo de identificação precoce dos problemas de leitura está descrita na Tabela 2.

Tabela 2

Distribuição da Média, Desvio Padrão, Valor Mínimo e Valor Máximo do Desempenho obtido pelos Escolares em Cada Prova do Protocolo de Identificação Precoce dos Problemas de Leitura em Situação de Pré-Testagem

\begin{tabular}{cccccc}
\hline Par de Variáveis & $n$ & Média & Desvio Padrão & Mínimo & Máximo \\
\hline CA & 60 & 20,98 & 2,85 & 12,00 & 23,00 \\
PR & 60 & 7,80 & 5,93 & 0,00 & 20,00 \\
IR & 60 & 12,87 & 5,91 & 0,00 & 20,00 \\
SS & 60 & 19,43 & 4,19 & 0,00 & 21,00 \\
PP & 60 & 18,12 & 3,59 & 7,00 & 22,00 \\
SF & 60 & 1,52 & 5,20 & 0,00 & 21,00 \\
AF & 60 & 4,85 & 5,92 & 0,00 & 20,00 \\
ISI & 60 & 6,45 & 7,06 & 0,00 & 21,00 \\
MT & 60 & 20,08 & 3,01 & 13,00 & 24,00 \\
AV & 60 & 9,35 & 1,22 & 4,00 & 10,00 \\
L & 60 & 22,40 & 15,54 & 0,00 & 40,00 \\
CF & 60 & 18,62 & 3,63 & 0,00 & 20,00
\end{tabular}

Nota. CA: conhecimento do alfabeto, PR: produção de rima, IR: identificação de rima, SS: segmentação silábica, PP: produção de palavras a partir do fonema dado, SF: síntese fonêmica, AF: análise fonêmica; ISI: identificação de som inicial, MT: memória de trabalho; AV: atenção visual, L: leitura, CF: compreensão de frases. Análise estatística: Teste dos Postos Sinalizados de Wilcoxon.

Os escolares que apresentaram valor mínimo inferior a $51 \%$ do valor máximo, em pelo menos 4 provas do Protocolo de identificação precoce dos problemas de leitura (conhecimento do alfabeto, consciência fonológica, me- mória de trabalho, nomeação rápida e leitura de palavras e pseudopalavras), em relação ao grupo-classe, foram considerados de risco para a dislexia, sendo submetidos ao programa de treinamento. Os escolares com desempenho 
superior a $51 \%$ nas provas do Protocolo não foram submetidos ao programa de treinamento por não apresentarem sinais de risco para a dislexia, constituindo, assim, o grupo controle deste estudo.

Dentre os 60 escolares (100\%) submetidos ao procedimento de identificação dos sinais da dislexia, 30 (50\%) apresentaram desempenho inferior. A partir desse achado, os escolares foram divididos em dois grupos: (a) Grupo I (GI): composto por 30 escolares submetidos ao programa de treinamento das habilidades fonológicas e correspondência grafema-fonema, sendo $30 \%$ do gênero masculino e $20 \%$ do gênero feminino. (b) Grupo II (GII): composto por 30 escolares não submetidos ao programa de treinamento das habilidades fonológicas e correspondência grafema-fonema, sendo $23 \%$ do gênero masculino e $27 \%$ do gênero feminino.

De maneira semelhante ao procedimento de avaliação utilizado neste estudo, foram elaboradas estratégias para o programa de treinamento das habilidades fonológicas e correspondência grafema-fonema, tomando-se como base a pesquisa original (Schneider et al., 2000). Esse programa foi composto por treze atividades trabalhadas em 18 sessões cumulativas, com duração de 50 minutos, realizadas em duas sessões semanais na escola de origem dos escolares. As atividades desenvolvidas no programa de treinamento estão descritas a seguir:

1. Identificação das letras e reconhecimento do alfabeto fonêmico. Foram apresentados ao escolar os 23 grafemas para identificar o nome e o valor sonoro de cada um.

2. Combinação de letra para a formação de sílabas e formação de palavras. Foi apresentado, nas sessões iniciais, um conjunto de quatro letras para o escolar combinar (consoante-vogal, consoante-consoantevogal), formando sílabas simples, sílabas complexas e posterior formação de palavras para a leitura. A partir da quarta sessão, o conjunto foi aumentado para seis letras.

3. Identificação de figuras pelo nome das letras. Foram apresentadas figuras para o escolar identificar qual delas começava com a letra visualmente apresentada.

4. Identificação de figuras pelo som das letras. Foram apresentadas figuras para o escolar identificar qual delas se iniciava com o som produzido pela pesquisadora.

5. Identificação de palavras dentro de uma frase. Foram apresentadas, auditivamente, sete frases afirmativas para o escolar dividir cada uma em palavras, marcando-as por palmas.

6. Identificação e manipulação de sílabas na palavra. Foram apresentadas, auditivamente, duas palavras para a identificação das sílabas iniciais e, em seguida, solicitado a manipulação de segmentação silábica para a formação de novas palavras em posição inicial, medial e final. Em cada sessão foram fornecidas seis palavras.
7. Síntese fonêmica. Foram apresentadas, auditivamente, sete palavras separadas por sons e solicitado ao escolar para reconhecer as palavras.

8. Rima. Foram solicitadas aos escolares palavras que terminassem com o mesmo som e que identificassem figuras que rimassem em sua nomeação.

9. Identificação e discriminação de fonemas. Foi apresentado auditivamente um fonema e solicitado ao escolar que mencionasse uma palavra que começasse com este som. Em seguida, foram apresentadas oralmente sete palavras e questionado se havia o fonema alvo em cada uma.

10. Segmentação de fonemas. Foi apresentada uma palavra oralmente e solicitado ao escolar para falar todos os fonemas dela. Nessa fase do programa, foram utilizadas fichas coloridas para auxiliá-lo a segmentar os sons das palavras auditivamente apresentadas. Em cada sessão foram fornecidas sete palavras.

11. Subtração de fonemas. Foram apresentadas ao escolar seis palavras oralmente para que retirasse o fonema final, e, em seguida, apresentadas seis palavras oralmente para que retirasse o fonema inicial.

12. Substituição de fonemas. Foi apresentada oralmente ao escolar uma palavra e solicitado que retirasse o fonema inicial e o substituísse por outro, formando novas palavras. Em cada sessão foram fornecidas sete palavras.

13. Transposição de fonemas. Foi solicitado ao escolar para falar as palavras em ordem inversa da palavra apresentada pela pesquisadora, formando novas palavras.

Depois de finalizado o programa de treinamento das habilidades fonológicas e correspondência grafemafonema, os escolares do GI e GII foram submetidos novamente à aplicação do Protocolo de identificação precoce dos problemas de leitura. Isso aconteceu após o período de 10 semanas de aplicação do programa de treinamento.

Ressalta-se que, em atenção aos aspectos éticos em pesquisa com seres humanos, os escolares de risco para a dislexia identificados no programa de treinamento foram encaminhados para diagnóstico interdisciplinar e tratamento fonoaudiológico no Centro de Estudos da Educação e Saúde da Universidade Estadual Paulista (CEES/ UNESP), caso, em situação de pós-testagem, não tivesse ocorrido melhora das habilidades avaliadas na primeira testagem.

Este estudo foi aprovado pelo Comitê de Ética em Pesquisa da Faculdade de Filosofia e Ciências da UNESP (CEP/FFC) sob o protocolo de $\mathrm{n}^{\circ} 3348 / 2008$.

Para a análise estatística, foi utilizado o Teste de Friedman, para verificar possíveis diferenças entre as variáveis do programa de treinamento das habilidades fonológicas e de correspondência grafema-fonema. Outro método de análise estatística utilizado foi a aplicação do Teste dos Postos Sinalizados de Wilcoxon, para verificar possíveis diferenças entre os momentos de pré 
Fukuda, M. T. M \& Capellini, S. A. (2012). Programa de Intervenção Fonológica Associado à Correspondência Grafema-Fonema em Escolares de Risco para a Dislexia.

e pós-testagem, considerados na avaliação de cada grupo. O nível de significância adotado para a aplicação dos testes estatísticos foi de $5 \%(0,050)$. A análise dos dados foi realizada utilizando o programa SPSS (Statistical Package for Social Sciences), em sua versão 17.0.

\section{Resultados}

A Tabela 3 apresenta a comparação do desempenho obtido pelo GI e pelo GII em situação de pré e póstestagem na realização do Protocolo de identificação precoce dos problemas de leitura.

Tabela 3

Distribuição da Média, Desvio Padrão e Valor de p do Desempenho obtido pelos Escolares do GI e GII em Situação de Pré e Pós-Testagem

\begin{tabular}{|c|c|c|c|c|c|c|c|}
\hline GI & Média & Desvio Padrão & Valor de $p$ & GII & Média & Desvio Padrão & Valor de $p$ \\
\hline CA_Pré & 20,10 & 3,29 & $<0,001 *$ & CA_Pré & 21,87 & 2,01 & 0,217 \\
\hline CA_Pós & 22,50 & 0,63 & & CA_Pós & 22,07 & 2,29 & \\
\hline PR_Pré & 4,70 & 4,34 & $<0,001 *$ & PR_Pré & 10,90 & 5,73 & $0,001 *$ \\
\hline PR_Pós & 17,33 & 3,32 & & PR_Pós & 13,67 & 4,23 & \\
\hline IR_Pré & 9,80 & 5,68 & $<0,001 *$ & IR_Pré & 15,93 & 4,38 & $0,009 *$ \\
\hline IR_Pós & 16,20 & 3,43 & & IR_Pós & 17,57 & 2,70 & \\
\hline SS_Pré & 18,13 & 5,64 & $0,004 *$ & SS_Pré & 20,73 & 0,58 & 0,053 \\
\hline SS_Pós & 20,57 & 1,41 & & SS_Pós & 20,97 & 0,18 & \\
\hline PP_Pré & 16,67 & 4,12 & $<0,001^{*}$ & PP_Pré & 19,57 & 2,21 & 0,103 \\
\hline PP_Pós & 20,93 & 0,25 & & PP_Pós & 20,27 & 1,41 & \\
\hline SF_Pré & 0,03 & 0,18 & $<0,001 *$ & SF_Pré & 3,00 & 7,11 & $0,019^{*}$ \\
\hline SF_Pós & 18,20 & 4,90 & & SF_Pós & 6,17 & 8,82 & \\
\hline AF_Pré & 1,80 & 3,08 & $<0,001^{*}$ & AF_Pré & 7,90 & 6,52 & $0,024 *$ \\
\hline AF_Pós & 17,77 & 4,32 & & AF_Pós & 10,93 & 8,25 & \\
\hline ISI_Pré & 3,23 & 3,57 & $<0,001 *$ & ISI_Pré & 9,67 & 8,20 & 0,844 \\
\hline ISI_Pós & 20,77 & 0,77 & & ISI_Pós & 9,53 & 8,07 & \\
\hline MT_Pré & 18,87 & 2,86 & $0,003 *$ & MT_Pré & 21,30 & 2,68 & 0,621 \\
\hline MT_Pós & 20,93 & 2,65 & & MT_Pós & 21,63 & 2,33 & \\
\hline VIF_Pré & 45,17 & 11,67 & $0,010^{*}$ & VIF_Pré & 34,07 & 5,53 & 0,053 \\
\hline VIF_Pós & 41,57 & 10,21 & & VIF_Pós & 31,80 & 5,65 & \\
\hline AV_Pré & 8,73 & 1,48 & $<0,001^{*}$ & AV_Pré & 9,97 & 0,18 & 0,317 \\
\hline AV_Pós & 9,77 & 0,43 & & AV_Pós & 10,00 & 0,00 & \\
\hline L_Pré & 11,47 & 12,37 & $<0,001^{*}$ & L_Pré & 33,33 & 9,55 & $0,017^{*}$ \\
\hline L_Pós & 27,80 & 9,04 & & L_Pós & 36,13 & 5,39 & \\
\hline CF_Pré & 17,77 & 4,84 & $0,004^{*}$ & CF_Pré & 19,47 & 1,38 & 0,931 \\
\hline CF_Pós & 19,87 & 0,57 & & CF_Pós & 19,63 & 1,00 & \\
\hline
\end{tabular}

Nota. CA: Conhecimento do alfabeto, PR: Produção de rima, IR: Identificação de rima, SS: Segmentação silábica, PP: Produção de palavra a partir do fonema dado, SF: Síntese fonêmica, AF: Análise fonêmica, ISI: Identificação do som inicial, MT: Memória de trabalho, VIF: Velocidade de acesso à informação fonológica, AV: Atenção visual, L: Leitura de palavras e não-palavras, CF: Compreensão de frases a partir de figuras, (GI): escolares submetidos à intervenção, (GII): escolares não submetidos à intervenção. Análise estatística: Teste dos Postos Sinalizados de Wilcoxon. 
Com a aplicação do Teste dos Postos Sinalizados de Wilcoxon, pode-se verificar que a média das habilidades cognitivo-linguísticas avaliadas do GII foi superior à do GI em situação de pré-testagem.

Após o período de dez semanas de realização do programa de treinamento, entretanto, no momento de póstestagem, aplicando o Teste dos Postos Sinalizados de Wilcoxon, os escolares do GI submetidos ao programa de treinamento evidenciaram diferença estatisticamente significante em todas as provas de habilidades cognitivolinguísticas.

Os escolares do GII apresentaram diferença estatisticamente significante nas provas de produção de rima (PR), identificação de rima (IR), síntese fonêmica (SF), análise fonêmica (AF) e leitura de palavras e não-pala$\operatorname{vras}(\mathrm{L})$.

Ao analisar o escore da média, em situação de pós testagem, verificou-se que os escolares do GI apresentaram desempenho superior em relação ao do GII nas provas de conhecimento do alfabeto (CA), produção de rima (PR), produção de palavra a partir do fonema dado (PP), síntese fonêmica (SF), análise fonêmica (AF), identificação do som inicial (ISI) e compreensão de frases a partir de figuras $(\mathrm{CF})$.

Em relação ao desempenho dos escolares do GI e GII em situação de pré e pós-testagem no subteste para a velocidade de acesso à informação fonológica foi possível observar que, no momento da pré-testagem, o escore médio do GI foi superior ao do GII, revelando maior tempo para acesso ao léxico de memória a curto prazo e nomeação fonológica, e que, em situação de pós-testagem, ocorreu um decréscimo nesse tempo, demonstrando uma diferença estatisticamente significante no GI. Os escolares do GII, entretanto, não evidenciaram diferença estatisticamente significante e mantiveram seu desempenho.

Dentre os 30 escolares (100\%) submetidos ao programa de treinamento de habilidades fonológicas e correspondência grafema-fonema, dois $(6,66 \%)$ continuaram apresentando valor mínimo inferior a $51 \%$ em 4 provas do Protocolo de identificação precoce dos problemas de leitura em relação ao grupo-classe em situação de póstestagem. Esses escolares foram encaminhados para a avaliação interdisciplinar, composta por avaliação neurológica e neuropsicológica, no CEES e, confirmado o quadro de dislexia, foram encaminhados para a intervenção fonoaudiológica no referido Centro.

\section{Discussão}

Este estudo enfatizou a identificação precoce da dislexia, levando em consideração que, na literatura (Gijsel, Bosman, \& Verhoeven, 2006; Landry, Swank, Smith, Assel, \& Gunnewig, 2006; Molfese et al. 2006), há evidências de que as habilidades cognitivas e lingüísticas, quando trabalhadas precocemente com escolares de risco para a dislexia influenciam a aquisição do princípio alfabético do sistema de escrita. A falta de resposta do escolar à intervenção seria um critério de diagnóstico precoce para a dislexia (Agnew et al., 2004; Elbro \& Petersen, 2004; Schneider et al., 2000).

Neste estudo, pode-se verificar que, dos 30 escolares considerados de risco para a dislexia, depois de serem submetidos ao programa de treinamento fonológico associado à correspondência grafema-fonema, 28 deles apresentaram melhora no desempenho em estratégias de percepção, discriminação, armazenamento e recuperação da informação fonêmica, corroborando o descrito em estudos internacionais (Etchepareborda, 2003; Harn, Linan-Thompson, \& Roberts, 2008; López-Escribano \& Beltrán, 2009; Schneider et al., 2000). Ocorreu, também, melhora de desempenho no grupo de escolares do grupo controle que não foram submetidos ao programa de treinamento, em um menor número de habilidades.

Esses resultados apontam para o fato de que quando os escolares recebem treinamento com relação às habilidades cognitivo-linguísticas, os resultados tornaram-se significativos nas provas fonológicas, de memória de trabalho e atenção visual, gerando impacto positivo na prova de leitura e velocidade de acesso à informação fonológica em relação aos escolares que não foram submetidos ao programa de treinamento, evidenciando que o uso de habilidades fonológicas que exigem para o seu processamento habilidades subjacentes, como atenção e memória, favorecem a aquisição do princípio alfabético da Língua Portuguesa. Esses achados confirmam estudos descritos na literatura (Cunha \& Capellini, 2009; Piasta, 2006; Share, 2004; Smythe et al., 2008; Stackhouse, 2004).

Assim, apesar de os escolares serem submetidos à mesma metodologia de alfabetização, este estudo evidenciou que a instrução explícita da consciência fonológica combinada à instrução da correspondência grafema-fonema deve ser ensinada para favorecer o desenvolvimento da leitura. Esses achados corroboram estudos descritos na literatura (Cunha \& Capellini, 2009; Piasta, 2006; Share, 2004; Smythe, et al., 2008; Stackhouse, 2004), podendose concluir que o ensino da base alfabética de uma língua auxilia o desenvolvimento da consciência fonológica, que, por sua vez, facilita a aquisição e desenvolvimento da leitura.

Apenas dois escolares não apresentaram melhora no desempenho em situação de pós-testagem depois de serem submetidos ao programa de treinamento das habilidades fonológicas e correspondência grafema-fonema, sendo confirmado o diagnóstico de dislexia pela realização de avaliação interdisciplinar. Verifica-se, então, que os 28 escolares apresentavam apenas falha no processo de alfabetização e não o quadro de dislexia. Esses achados remetem à reflexão de que os sinais descritos na literatura nacional e na internacional (American Speech-LanguageHearing Association, 2003; Etchepareborda, 2003; LópezEscribano \& Beltrán, 2009) sofrem interferência direta da metodologia de ensino, o que justifica o fato de os fonoaudiólogos, juntamente com os professores, utilizarem programas de intervenção com base fonológica e 
relação grafema-fonema para identificar e intervir precocemente nos sinais da dislexia e, consequentemente, diminuir o número de encaminhamentos desnecessários para a realização de diagnóstico fonoaudiológico. Essa prática corrobora estudos descritos na literatura internacional (Broom \& Doctor, 1995; Calhoon, 2005; Fletcher, Coulter, Reschly, \& Vaughn, 2004; Harn et al., 2008; López-Escribano, 2007; Ryder, Tunmer, \& Greaney, 2008; Simmons, Coyne, McDonagh, Harn, \& Kame'enui, 2008; Simmons et al., 2007; Simos et al., 2007).

A adaptação deste programa enfocou a atenção, percepção, discriminação e memória auditiva e visual de forma direta em todas as sessões, proporcionando aos escolares submetidos ao programa melhor e maior tempo de atenção à base fonológica do sistema de escrita da Língua Portuguesa. Um problema de percepção da fala pode causar um efeito em cascata, iniciando com o rompimento do desenvolvimento normal do sistema fonológico e resultando em problemas na aprendizagem da leitura e da soletração (Boets, Wounters, Van Wieringen, \& Ghesquière, 2007).

Assim, os achados deste estudo apresentam o treinamento da habilidade fonológica e correspondência grafema-fonema em escolares de risco para a dislexia como um instrumento de auxílio ao diagnóstico de uma condição determinada genética e neurologicamente, como a dislexia. Conforme descrição na literatura internacional (Elbro \& Petersen, 2004; Gijsel et al., 2006; Landry et al., 2006; Schneider et al., 2000; Vaughn et al., 2006), o fracasso do escolar de risco a este tipo de treinamento é um critério diagnóstico para a dislexia. Esses achados apontam para o fato de que quanto menor for a instrução na base fonológica e na relação grafema-fonema para a aprendizagem da leitura, maior será o risco de identificação errônea de um escolar como sendo disléxico.

\section{Conclusão}

Os achados deste estudo permitiram concluir que os escolares com sinais de risco para a dislexia, depois de serem submetidos ao programa de treinamento das habilidades fonológicas e correspondência grafema-fonema, apresentaram melhora no desempenho em situação de pós-testagem em comparação com a pré-testagem, superando, em algumas provas, a média de desempenho dos escolares não submetidos ao programa de treinamento. Esse fato evidencia a eficácia do programa do treinamento utilizado e a resposta à intervenção como um critério de diagnóstico para a identificação precoce da dislexia.

Os dados referentes ao Protocolo de identificação precoce dos problemas de leitura em situações de pré e pós testagem evidenciaram a importância da realização de uma avaliação que enfoque as habilidades fonológicas e a relação letra-som no âmbito clínico e educacional, visto que isso auxiliaria o escolar em situação de alfabetização a desenvolver habilidades cognitivo-linguísticas necessárias para a aprendizagem da base alfabética do sistema de escrita do português brasileiro. Por meio dessa instrução formal seria possível garantir que os escolares que não apresentassem aprendizagem do mecanismo de relação letra-som fossem eletivos para o diagnóstico da dislexia. E, ainda, que a distinção entre o mau leitor e o leitor disléxico poderia ser finalmente reconhecida entre os profissionais que atuam no diagnóstico e intervenção dos problemas de aprendizagem.

Dessa forma, a melhora nas habilidades fonológicas apresentadas pelos escolares submetidos ao programa de treinamento aponta para a necessidade de uma reflexão sobre a metodologia de alfabetização utilizada em situação de sala de aula, já que as habilidades de atenção, discriminação e percepção dos sons e letras trabalhadas proporcionaram aumento na sensibilidade e percepção fonológica, o que se refletiu também na melhora do tempo de realização da leitura, evidenciando que os escolares considerados de risco, em sua maioria, não apresentavam o quadro de dislexia.

\section{Referências}

Abreu, M. de, \& Cardoso-Martins, C. (1998). Alphabetic access route in beginning reading acquisition in Portuguese: The role of letter-name knowledge. Reading and Writing: An Interdisciplinary Journal, 10(2), 85-104.

Agnew, J. A., Dorn, C., \& Eden, G. F. (2004). Effect of intensive training on auditory processing and reading skills. Brain Language, 88(1), 21-25.

American Speech-Language-Hearing Association. (2003). Language-based learning disabilities. Retrieved August 29, 2003, from http://www.asha.org

Boets, B., Wounters, J., van Wieringen, A., \& Ghesquiére, P. (2007). Auditory processing, speech perception and phonological ability in pre-school children at high-risk for dyslexia: A longitudinal study of the auditory temporal processing theory. Neuropsychologia, 45(8), 1608-1620.

Broom, Y. M., \& Doctor, E. A. (1995). Developmental phonological dyslexia: A case study of the efficacy of a remediation programme. Cognitive Neuropsychology, 12(7), 725-766

Calhoon, M. B. (2005). Effects of a peer-mediated phonological skill and reading comprehension program on reading skill acquisition for middle school students with reading disabilities. Journal Learning Disabilities, 38(5), 424-433.

Capellini, S. A., \& Ciasca, S. M. (2000). Avaliação da consciência fonológica em criança com distúrbio específico da leitura e escrita e distúrbio de aprendizagem. Temas sobre Desenvolvimento, 8(48), 17-23.

Capellini, S. A., Padula, N. A. M. R., \& Ciasca, S. M. (2004). Desempenho de escolares com distúrbio específico de leitura em programa de remediação. Pró-Fono Revista de Atualização Científica, 16(3), 261-274.

Capellini, S. A., Sampaio, M. N., Fukuda, M. T. M., Oliveira, A. M., Fadini, C. C., \& Martins, M. A. (2009). Protocolo de identificação precoce dos problemas de leitura: Estudo preliminar com escolares de $1^{\circ}$ ano escolar. Revista Psicopedagogia, 26(81), 367-375. 
Capellini, S. A., Sampaio, M. N., Kawata, K. H. S., Padula, N. A. M. R., Santos, L. C. A., Lorencetti, M. D., et al. (2010). Eficácia terapêutica do programa de remediação fonológica em escolares com dislexia do desenvolvimento. Revista CEFAC, 12(1), 27-39.

Capellini, S. A., \& Salgado, C. A. (2003). Avaliação fonoaudiológica do distúrbio específico de leitura e distúrbio de aprendizagem: Critérios diagnósticos, diagnóstico diferencial e manifestações clínicas. In S. M. Ciasca (Ed.), Distúrbio de aprendizagem: Proposta de avaliação interdisciplinar (pp. 141-164). São Paulo, SP: Casa do Psicólogo.

Critchley, M. (1985). Specific developmental dyslexia. In J. A. M. Frederiks (Ed.), Handbook of Neurology (pp. 353-364). Amsterdam, The Netherlands: Elsevier.

Cunha, V. L. O., \& Capellini, S. A. (2009). Desempenho de escolares de $1^{\mathrm{a}}$. a $4^{\mathrm{a}}$. série do ensino fundamental nas provas de habilidades metafonológicas e de leitura-PROHMELE. Revista Sociedade Brasileira Fonoaudiologia, 14(1), 56-68.

Elbro, C., \& Petersen, D. K. (2004). Long-term effects of phoneme awareness and setter sound training: An intervention study with children at risk for dyslexia. Journal Educational Psychology, 96(4), 660-670.

Etchepareborda, M. C. (2003). La intervención en los trastornos disléxicos: entrenamiento de la conciencia fonológica. Revista Neurology, 36, 13-19.

Fletcher, J. M., Coulter, W. A., Reschly, D. J., \& Vaughn, S. (2004). Alternative approaches to the definition and identification of learning disabilities: Some questions and answers. Annals Dyslexia, 54(2), 304-331.

Gijsel, M. A. R., Bosman, A. M. T., \& Verhoeven, L. (2006). Kindergaten risk factors, cognitive factors, and teacher judgments as predictors of early reading in Dutch. Journal Learning Disability, 39(6), 558-571.

Harn, B. A., Linan-Thompson, S., \& Roberts, G. (2008). Intensifying instruction: Does additional instructional time make a difference for the most at-risk first graders? Journal Learning Disabilities, 41(2), 115-125.

Landry, S. H., Swank, P. R., Smith, K. E., Assel, M. A., \& Gunnewig, S. B. (2006). Enhancing early literacy skills for preschool children: Bringing professional development model to scale. Journal Learning Disabilities, 39(4), 306-324.

López-Escribano, C. (2007). Contribuciones de la neurociencia al diagnóstico y tratamiento educativo de la dislexia del desarrollo. Revista de Neurología, 44(3), 173-180.

López-Escribano, C., \& Beltrán, J. A. (2009). Early predictors of reading in three groups of native Spanish speakers: Spaniards, Gypsies, and Latin Americans. Spanish Journal of Psychology, 12, 84-95.

Marinotti, M. (2004). Processos comportamentais envolvidos na aprendizagem da leitura e da escrita. In M. M. C. Hubner \& M. Marinotti (Eds.), Análise do comportamento para a educação: Contribuições recentes (pp. 205-240). Santo André, SP: ESETec.

Molfese, V. J., Modglin, A. A., Beswick, J. L., Neamon, J. D., Berg, S. A., Berg, C. J., et al. (2006). Letter knowledge, phonological processing, and print knowledge: Skill development in non-reading preschool children. Journal Learning Disabilities, 39(4), 296-305.

Piasta, S. B. (2006). Acquisition of alphabetic knowledge: Examining letter- and child-level factors in a single, comprehensive model. (Unpublished master's thesis). Florida State University, FL.
Ryder, J. F., Tunmer, W. E., \& Greaney, K. T. (2008). Explicit instruction in phonemic awareness and phonemically based decoding skills as an intervention strategy for struggling readers in whole language classrooms. Reading and Writing, 21, 349-369.

Schneider, W., Roth, E., \& Ennemoser, M. (2000). Training phonological skills and letter knowledge in children at risk for dyslexia: A comparison of three kindergarten intervention programs. Journal Educational Psychology, 92(2), 284-295.

Scliar-Cabral, L. (2003). Princípios do sistema alfabético do português do Brasil. São Paulo, SP: Contexto.

Share, D. L. (2004). Knowing letter names and learning letter sounds: A causal connection. Journal Experimental Child Psychology, 88, 213-233.

Simmons, D. C., Coyne, M. D., McDonagh, S., Harn, B. A., \& Kame'enui, E. J. (2008). Indexing response to intervention: A longitudinal study of reading risk from kindergarten through third grade. Journal Learning Disabilities, 41(2), 158-173.

Simmons, D. C., Kame'enui, E. J., Harn, B., Coyne, M. D., Stoolmiller, M., Santoro, L. E., et al. (2007). Attributes of effective and efficient kindergarten reading intervention: An examination of instructional time and design of instruction specificity. Journal Learning Disabilities, 40, 331-347.

Simos, P. G., Sarkari, S., Billingsley, R. L., Fletcher, J. M., Denton, C., \& Papanicolaou, A. C. (2007). Altering the brain circuits for reading through intervention: A magnetic source imaging study. Neuropsychology, 21(4), 485-486.

Smythe, I., Everatt, J., Al-Menaye, N., He, X., Capellini, S. A., Gyarmathy, E., et al. (2008). Predictors of world-level literacy amongst grade 3 children in five diverse languages. Dyslexia, 14(3), 170-187.

Snow, C. E., \& Juel, C. (2005). Teaching children to read: What do we know about how to do it? In M. J. Snowling \& C. Hulme (Eds.), The science of reading: A handbook (pp. 501520). Oxford, UK: Blackwell.

Stackhouse, J. (2004). Fala, ortografia e leitura: Quem está em risco e por quê? In M. Snowling \& J. Stackhouse, Dislexia, fala e linguagem: Um manual do profissional (pp. 280-324). Porto Alegre, RS: Artmed.

Temple, E., Poldrack, R. A., Protopapas, A., Nagarajan, S., Salz, T., Tallal, P., et al. (2000). Disruption of neural response to rapid acoustic stimuli in dyslexia: Evidence from functional MRI. Proceedings of the National Academy of Sciences of the United States of America, 97(25), 13907-13912.

Vaughn, S., Linan-Thompson, S., Mathes, P. G., Cirino, P. T., Carlson, C. D., Pollard-Durodola, S. D., et al. (2006). Effectiveness of Spanish intervention for first-grade English language learners at risk for reading difficulties. Journal Learning Disabilities, 39(1), 56-73.
Recebido: 05/08/2010 $1^{a}$ revisão: 02/05/2011 Aceite final: 10/10/2011 